\title{
A nanoliter fluidic platform for large-scale single nucleotide polymorphism genotyping
}

\author{
Douglas G. Roberts, Tom B. Morrison, Shau Neen Liu-Cordero, Jamie Cho, Javier Garcia, Tanya S. Kanigan, \\ Kevin Munnelly, and Colin J.H. Brenan \\ BioTrove Inc., Woburn, $M A$, USA
}

BioTechniques 46:Pix-Pxiii (BioTechniques for Preclinical Development March 2009) doi 10.2144/000112887

Keywords: 5'-exonuclease assay; TaqMan PCR; SNP genotyping; nanofluidics; high-throughput genotyping; nanoliter PCR

Supplementary material for this article is available at www.BioTechniques.com/article/112887.

Discovery, evaluation, and understanding the biological relevance of single nucleotide polymorphisms (SNPs) and their associated phenotypes is relevant to many applications, including human disease diagnostics, pathogen detection, and identification of genetic traits impacting agricultural practices, both in terms of food quality and production efficiency. Validation of putative SNP associations in large-scale cohorts is currently impeded by the technical challenges and high cost inherent in analyzing large numbers of samples using available SNP genotyping platforms. We describe in this report the implementation of the $5^{\prime}$-exonuclease, biallelic PCR assay for SNP genotyping (TaqMan) in a nanofluidic version of a high-density microplate. System performance was assessed using a panel of 32 TaqMan SNP genotyping assays targeted to human polymorphisms. This functional test of the nanoliter fluidic SNP genotyping platform delivered genotyping call rates and accuracies comparable to the same larger volume reactions in microplate systems.

\section{Introduction}

Single nucleotide polymorphisms (SNPs) are the most abundant and accessible class of biallelic polymorphisms for recording genetic variation in a population and offer considerable advantages over highly polymorphic, repetitive loci (i.e., short tandem repeats and microsatellites) because of their lower mutation rates and nearly uniform genomic distribution. The popularity of SNP genotyping has increased with the emergence and widespread availability of technologies for whole-genome SNP identification (1-4) and has resulted in a growing number of SNP databases for a wide range of organisms, including humans (www.ncbi.nlm.nih.gov/projects/ SNP). The shifting focus from discovery to utilization of SNP genotypes for cohort phenotype classification and segmentation has led to applications in human disease diagnostics $(5,6)$; pharmacogenetics $(7,8)$; blood typing $(9,10)$; DNA fingerprinting for criminal or parental verification (11); fisheries management $(12,13)$; plant and human pathogen detection and identification $(14,15)$; and marker-assisted crop and animal breeding (16-18).

A number of strategies have been proposed for scaling SNP genotyping to sample throughputs in the range of hundreds to thousands of specimens and analyzed with focused sets of ten to hundreds of predictive SNPs $(19,20)$. Here, we describe an alternative high-throughput genotyping system based on a highly parallel implementation of well-established end-point TaqMan PCR SNP genotyping assays in a miniaturized nanofluidic system. TaqMan (Applied Biosystems, Foster City, CA, USA) is a homogenous, biallelic PCR assay utilizing $5^{\prime}$-hydrolysis of two different dark quenched fluorescent probes (21) to distinguish between SNPs on one or both alleles. Each TaqMan SNP assay used in this study is labeled with VIC, a fluorescent dye from Applied Biosystems, and 5-carboxyfluorescein (FAM). The combined specificity of PCR primer and probe leads to a high genotyping accuracy and call rate.

Although there have been numerous reports of PCR micro- and nanofluidic systems (22), here we employ a nanotiter plate, a configuration that readily scales to higher genotyping throughputs without the need for advanced automation and that avoids the significantly higher reagent consumption of PCR in microtiter plates $(23,24)$. Previously described for use in SYBR Green-based real-time PCR measurement of gene expression levels (25), the nanotiter plate is similar in concept to a microtiter plate whereby a rectilinear array of 3072 through-holes grouped in 48 subarrays of 64 through-holes each is micromachined in a stainless steel platen the size of a microscope slide (26-28) (Figure 1). Each through-hole supports implementation of an independent $33 \mathrm{~nL}$ PCR assay, thus enabling a large number of PCR measurements to be made simultaneously on one or more samples.
One advantage of genotyping a specimen with multiple, independent TaqMan PCR assays is that each assay in an assay panel can be designed and optimized under a uniform set of conditions for maximum sensitivity and specificity. Furthermore, if the SNP panel changes in the future, then assays can be independently replaced without the need to re-optimize the entire assay set.

\section{Materials and methods}

Nanotiter plate system

The nanotiter plate, which is commercially available as the OpenArray system (BioTrove Inc., Woburn, MA, USA), features a stainless steel (317 stainless steel) platen the size of a microscope slide $(25 \mathrm{~mm}$ $\times 75 \mathrm{~mm} \times 0.3 \mathrm{~mm}$ ) etched to form a rectilinear array of 3072 holes with diameter 320 $\mu \mathrm{m}$, which are grouped in 48 subarrays of 64 through-holes each. The interior surface of each etched hole is made hydrophilic and the exterior surface of the plate is made hydrophobic by covalently linked polymer coatings. Wetting of the hydrophilic surface wicks fluid into each hole and capillary forces act against gravitational and inertial forces to retain the liquid in each hole in isolation.

\section{TaqMan SNP assays}

TaqMan SNP assays were dispensed into microplates for transfer into a nanotiter plate at a final $1 \times$ concentration by an automated, four-axis robotic fluid transfer 
system. This system is based on an array of 48 slotted pins in which each pin transfers a 33-nL aliquot of assay reagent from one well in the source plate to multiple holes in the nanotiter plate. Assay solvent is evaporated under controlled environmental conditions and the loaded plates are stored at $-20^{\circ} \mathrm{C}$ in a vacuum-sealed Mylar bag. Thirty-two TaqMan assays were deposited in duplicate in 64 through-holes of a subarray. A list of the TaqMan SNP assays and CEPH DNA samples used in the study are found in Supplementary Tables 1 and 2, respectively.

\section{DNA samples and PCR mix}

PCR reactions were prepared in MatriCal 384-well plates (Cat. no. MCR-101-1-2; Spokane, WA, USA) using a total volume of $5 \mu \mathrm{L}$ per well (corresponding to $5 \mu \mathrm{L}$ for each of the 48 subarrays in a single nanotiter plate) and a final concentration of $1 \times$ Universal Master Mix (Cat. no. 4324018; Applied Biosystems), 0.5\% Glycerol (Sigma, St. Louis, MO, USA), 1\% Pluronic F38 (BASF; Florham Park, NJ, USA) and $50 \mathrm{ng} /$ $\mu$ L BSA (Cat. no. A7096, Sigma). Ninety CEPH DNA samples (Coriell, Camden, NJ, USA) were purchased and concentrated to a starting concentration of $75 \mathrm{ng} /$ $\mu \mathrm{L}$. For the DNA titration experiment, the following final concentrations of DNA after mixing with the PCR reagents were used: $30.3 \mathrm{ng} / \mu \mathrm{L}, 15.1 \mathrm{ng} / \mu \mathrm{L}, 7.6 \mathrm{ng} / \mu \mathrm{L}, 3.0 \mathrm{ng} /$ $\mu \mathrm{L}, 1.5 \mathrm{ng} / \mu \mathrm{L}$, and $0.3 \mathrm{ng} / \mu \mathrm{L}$ (resulting in final DNA amounts of $1 \mathrm{ng}, 500 \mathrm{pg}, 250 \mathrm{pg}$, $100 \mathrm{pg}, 50 \mathrm{pg}$, and $10 \mathrm{pg}$ per 33-nL throughhole, respectively). All experiments used $90 \mathrm{CEPH}$ DNA templates along with six no template controls (NTCs) except where noted otherwise.

Aliquots of the DNA-PCR reagent mixture were transferred by capillary action into pipette tips within a 48 -tip array. The loaded pipette tips were placed in contact with the nanotiter plate within a two-axis automated motion system and positioned such that each pipette tip addressed a single subarray. Fluid was transferred into each through-hole of a subarray by moving the pipette tip array over a prescribed rectilinear path relative to the nanotiter plate. Wetting of the interior hydrophilic surface by the fluid retained in the pipette tips draws fluid into each through-hole and the hydrophobic exterior surface ensures fluidic isolation of each through-hole.

Thermal cycler protocol and imaging The platen was inserted into a glass-walled, slotted cassette filled with an immiscible perfluorinated liquid (Fluorinert; 3M, St. Paul, MN, USA) and hermetically sealed with a plug of UV-cured epoxy. Thermal cycling of the nanotiter plates was performed on a MJ Research PTC-200 peltier thermal cycler equipped with a dual tower for the simultaneous thermal cycling of 32 nanotiter
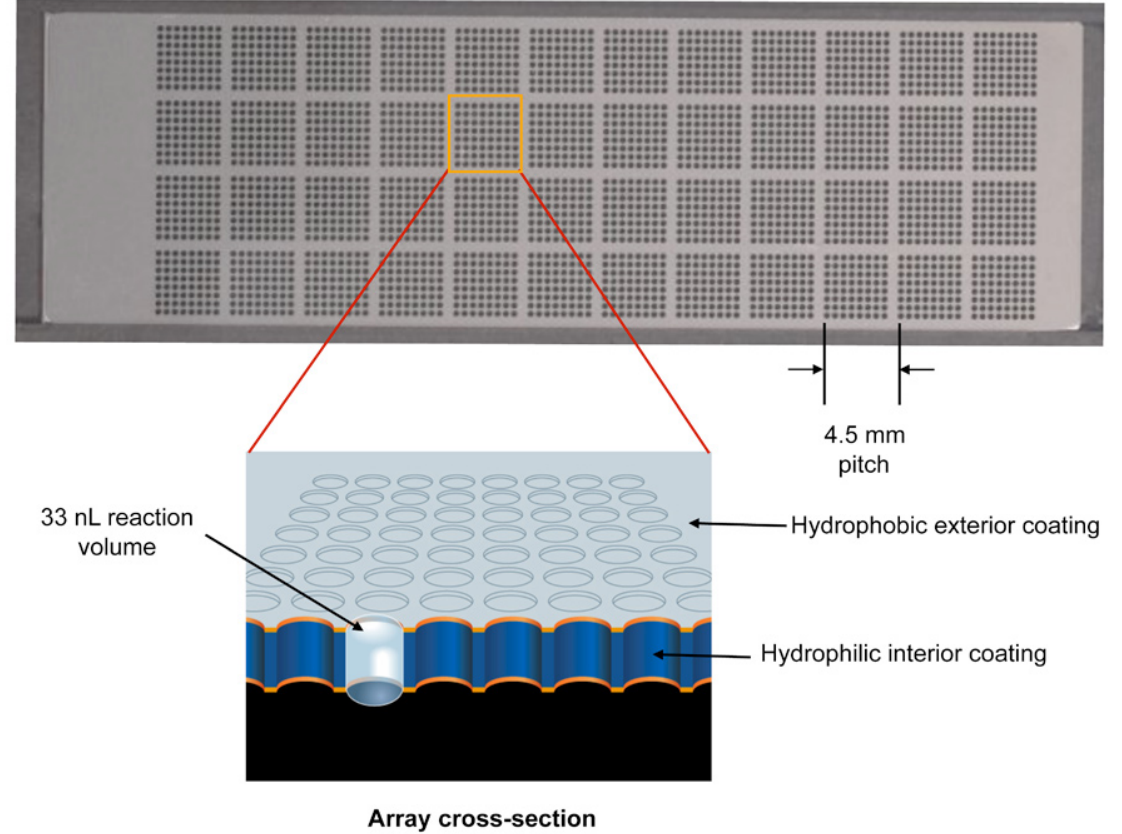

Figure 1. A stainless steel platen (317 stainless steel) the size of a microscope slide $(25 \mathrm{~mm} \times 75$ $\mathrm{mm} \times 0.3 \mathrm{~mm}$ ) is photolithographically patterned and wet-etched to form a rectilinear array of $\mathbf{3 0 7 2}$ micro-machined, $\mathbf{3 2 0} \mu \mathrm{m}$ diameter holes of $\mathbf{3 3} \mathrm{nL}$ each. The 48 groups of 64 holes are spaced at 4.5 $\mathrm{mm}$ to match the pitch of the wells in a 384-well microplate. A PCR compatible poly(ethylene glycol) hydrophilic layer is amine-coupled to the interior surface of each hole and a hydrophobic fluoroalkyl layer is vinyl-coupled to the exterior surface of the platen, resulting in the retention in individual, isolated containers of TaqMan PCR reagents and sample introduced into the nanotiter plate.

A

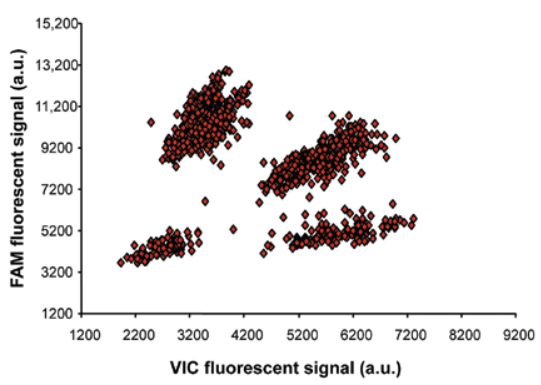

B

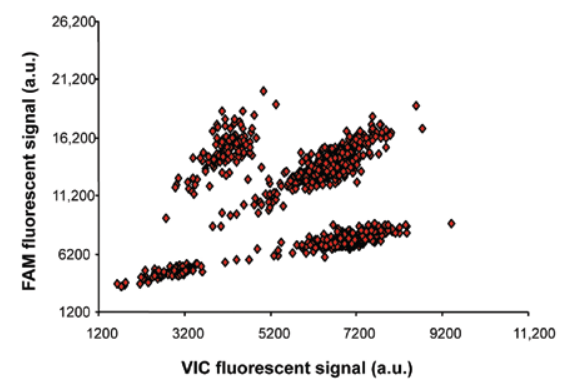

Figure 2. Overlay of SNP genotype data for 1216 samples and 2 TaqMan PCR assays from 19 nanotiter plates taken from 4 different manufacturing runs. Samples were 90 CEPH DNAs and 6 NTCs with 1 ng of DNA per reaction. (A) Assay C__-1290186_10, call rate $=98.88 \%$, accuracy $=99.9 \%$; (B) Assay C___1913912_10, call rate $=99.9 \%$, accuracy $=99.9 \%$. a.u., arbitrary units.

plates (Waltham, MA, USA). The thermal cycle protocol started with a $10-\min 91^{\circ} \mathrm{C}$ polymerase activation step followed by 50 cycles of $23 \mathrm{~s}$ at $51^{\circ} \mathrm{C}, 30 \mathrm{~s}$ at $53.5^{\circ} \mathrm{C}, 13 \mathrm{~s}$ at $54.5^{\circ} \mathrm{C}, 22 \mathrm{~s}$ at $97^{\circ} \mathrm{C}$, and $7 \mathrm{~s}$ at $92^{\circ} \mathrm{C}$. After temperature cycling, the nanotiter plates were held at $20^{\circ} \mathrm{C}$ for $5 \mathrm{~min}$ before removal for imaging. The nanotiter plates were then imaged in groups of three in an NT Imager (BioTrove Inc.), which is comprised of paired off-axis high-energy light-emitting diode (LED) excitation sources, a computercontrolled emission filter wheel, and a thermoelectrically cooled CCD camera (Model no. ST-10XMEI, SBIG, Santa Barbara, CA, USA). The LED emission wavelengths coincided with the maximum absorption wavelength for FAM and VIC dyes, which have excitation/emission fluorescent dye wavelengths of 495/520 nm and $538 / 554 \mathrm{~nm}$, respectively. Instrument software automatically captured and processed fluorescent images of the nanotiter plates to measure the fluorescent signal from each through-hole, and displayed the data as cluster plots for manual or semi-automated genotype calling by the user.

Whole-genome amplification method $90 \mathrm{CEPH}$ DNA templates were diluted to the minimum required concentration of $10 \mathrm{ng} / \mu \mathrm{L}$. They were then amplified with 
the GenomiPhi V2 DNA Amplification Kit (GE Healthcare, Piscataway, NJ, USA) following the manufacturer's instructions. Amplification was confirmed by agarose gel electrophoresis and quantities were verified with a SpectraMax Plus spectrophotometer (Molecular Devices, Sunnyvale, CA, USA). Samples were then diluted to manufacturerrecommended concentrations and run with the standard protocol for TaqMan SNP assays in the nanotiter plate with 32 assays in duplicate.

Genotype calling: expected genotypes Expected genotype calls for all templates for 19 of the 32 assays used in this study were obtained from the Single Nucleotide Polymorphism Database (dbSNP) (www.ncbi.nlm.nih.gov/projects/SNP). For the remaining assay/template combinations where no external verification of the expected genotype could be made, an expected call was generated by selecting the most frequently obtained call using a data set containing a minimum of 20 replicates.

Genotype calling: general considerations and exclusive strategy Genotype assignments for single data points for each assay and template were made using the Open Array SNP Genotyping Software v1.0.17.0 (BioTrove Inc.). The software functions by plotting a scatter plot of the FAM and VIC signals for both reporter dyes for each assay. The user selects a rectangular region (the exclusion box) from the origin to a user-defined location to exclude low signal points near the origin (e.g., fluidic errors, no template controls). The user then selects a point for the clustering origin and the cluster center for each of the three clusters. The intersection of lines uniformly bisecting each cluster is the clustering axis which, if applied correctly, will bisect the long axis of the cluster. Calls are assigned to each data point based on the number of standard deviations the point is away from the nearest cluster line and the other cluster lines. Calls may be further refined by the application of two threshold metrics: stringency and tolerance. The stringency is the number of standard deviations away from the nearest neighboring cluster line, and is used to improve the cluster variance estimate by allowing the user to remove outliers prior to final cluster variance calculation. Tolerance is the number of standard deviations away from the nearest neighboring cluster line and allows data points too near neighboring clusters to be classified as no calls. For an exclusive calling strategy, the exclusion box is typically set abutting the lower edge of the heterozygote cluster. The stringency and tolerance must be refined for each assay individually, but are generally refined in such a way as to exclude data points
A

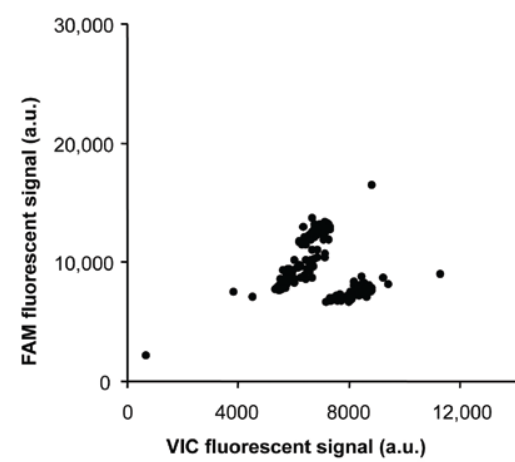

B

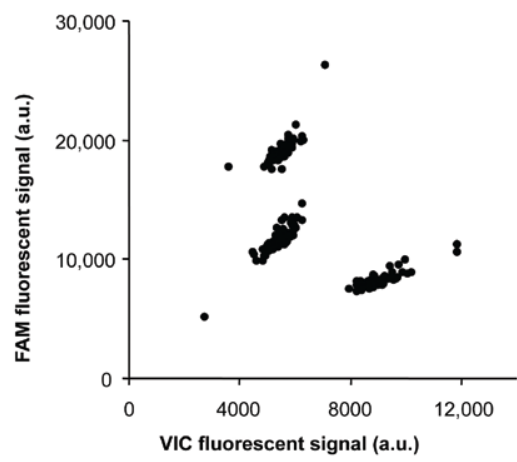

Figure 3. Example of improved genotype cluster separation through probe redesign. Plots indicate clusters from original Assay-By-Design (A) and an assay differing by a single additional nucleotide to the $5^{\prime}$ end of probe (B). a.u., arbitrary units.

residing between clusters for which an actual genotype call would be ambiguous.

Genotype calling: inclusive strategy

The inclusive strategy for genotype calling uses the same general idea as the exclusive strategy except that the aim of the inclusive strategy is to make genotype calls for as many data points as possible. The exclusion box is typically set somewhere between the origin and the lower edge of the heterozygote cluster. Typically, this set point is placed by the user at the approximate halfway point between the origin and the lower boundary of the heterozygote cluster, but encompassing any NTC. Stringency and tolerance thresholds are adjusted to allow all points outside the exclusion box to be called. The inclusive strategy is used for making calls with duplicates.

\section{Genotype calling: dupilcate call} assignment

Duplicate call evaluation was made using the following procedure for a pair of assay/ template replicates: For any pair of calls where both individual calls are no calls, a no call was assigned to both replicates. For any pair where one is not called and the other replicate has an assigned call, the assigned call is applied to both replicates. For any pair where two calls are made, and the calls are different, a no call is assigned to both replicates. For any pair where both replicates are called and the calls agree, the call is allowed to remain for both replicates of the pair.

For the determination of call rate and accuracy for the evaluation of either duplicate or single calls, the following equations were used:

$$
\% \text { Call rate }=\left(\frac{\text { Total possible calls }- \text { no calls }}{\text { Total possible calls }}\right) \times 100
$$

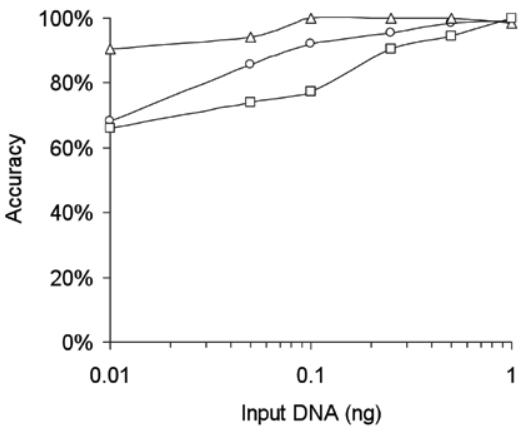

Figure 4. Effect of DNA concentration on PCR Array TaqMan SNP assay accuracy. Plot indicates average accuracy for assays C___8948116_1_ (square) and C___2153793_10 (triangle), or combined results from 24 different assays (circle) at indicated starting DNA quantity.

$\%$ Accuracy $=\left[100-\left(\frac{\text { Miscalls }}{\text { Miscalls }+ \text { correct calls }}\right)\right] \times 100$

\section{Results}

To evaluate the reproducibility of TaqMan SNP genotyping using this platform, the genotyping accuracy and miscall rate of 32 different TaqMan SNP assays were assessed by testing $90 \mathrm{CEPH}$ DNA samples and determining concordance with genotypes in dbSNP. These TaqMan assays were selected as representative of the range of assay performances we have encountered in the course of implementing over a thousand different Applied BioSystems Human TaqMan predesigned SNP genotyping assays (Cat. no. 4351379$)$ in the nanotiter plate system. Fourteen replicate runs were performed, each on a set of two nanotiter plates from different manufacturing lots. These 28 nanotiter plates (two nanotiter plates per sample set) generated a data set of 86,016 genotypes. Assays were considered to have 
A

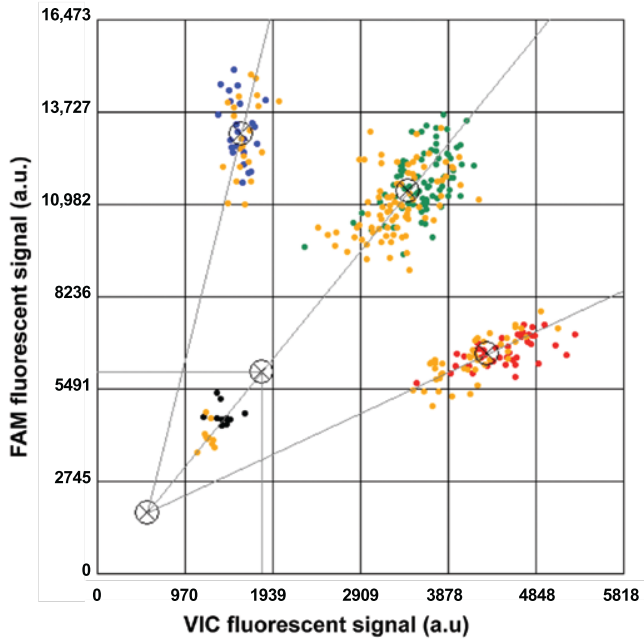

B

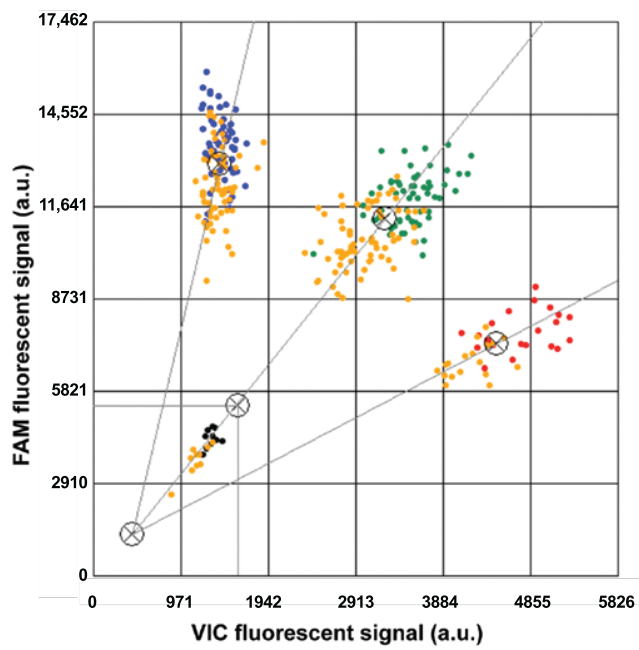

Figure 5. SNP genotyping with low DNA concentration. Ninety-six CEPH DNAs were split into two separate pools of $30 \mathrm{ng} / \mu \mathrm{L}$ final concentration. The first pool was diluted by a factor of 10 and pre-amplified with a commercial whole-genome amplification kit resulting in a $448 \mathrm{ng} / \mu \mathrm{L}$ concentration prior to genotyping with a panel of 32 TaqMan SNP assays in duplicate. The second unamplified pool was genotyped with the same assay panel under the same conditions. Panel A overlays the genotype results of one SNP genotype assay from the assay panel applied to the amplified and unamplified sample pools. Panel B shows the genotype results of a different SNP assay applied to the same sample pools. Data points correspond to the two homozygote genotypes (blue, red), the heterozygote genotype (green), the no template control (NTC) (black) for the unamplified DNA samples, and the WGA amplified samples (orange). Samples that fall within the gray box demarcated by the $\otimes$ symbols are not called. In all cases the call rate was $99 \%$ and accuracy was equal to $100 \%$. a.u., arbitrary units.

shown acceptable performance if they demonstrated $>95 \%$ call rate and $>99.5 \%$ accuracy based on reference to the dbSNP database. In the nanotiter plate, 27 of 32 assays in singleton met these criteria. $0.5 \%$ $(n=408)$ of data points were lost because of fluidics and formatting errors, $1.4 \%$ $(n=1074)$ of the remaining data points were not called because they could not be associated with a particular cluster, and, of the remaining called samples, $0.4 \%(n=$ $275)$ were miscalled, resulting in an overall accuracy of $99.6 \%(n=76,554)$. Duplicate calling (see Materials and Methods) increased the call accuracy to $99.9 \%$ with a slight decrease in call rate to $96.3 \%$.

To test the TaqMan assay concordance between the nanotiter plate and a 384-well microplate, the same 32 SNP assays were performed using the same $90 \mathrm{CEPH}$ DNA samples, but this time in a $5-\mu \mathrm{L}$ reaction volume using an ABI Prism 7900 thermocycler (Cat. no. 4329001; Applied Biosystems); results were called in singleton. 25 of 32 assays passed the $>95 \%$ call-rate performance criteriaand $>99.5 \%$ callaccuracy referenced to dbSNP. Interestingly, the assays that failed the accuracy and call criteria were different between the micro- and nanotiter plate, suggesting that $5-10 \%$ of the assays were sensitive to the PCR environment. This is not surprising since TaqMan assays are designed and optimized in thermoplastic microplates in microliter reaction volumes, a different thermodynamic environment from the nanoliter reaction volumes of the metal nanotiter plate.

Figure 2 shows reproducibility of SNP genotype data across four different manufacturing lots with 1216 samples consisting of $90 \mathrm{CEPH}$ DNA templates and six NTCs genotyped with 32 TaqMan assays in 19 nanotiter plates. Analysis of the data set found $>98.88 \%$ genotype call rate and accuracy for each assay.

There are several ways of optimizing TaqMan assay performance in the nanotiter plate. The first is a duplicate-calling strategy where duplicates of the affected assays require exact agreement to make a genotype call. Under these conditions, four previously failing assays passed the accuracy and call rate threshold, whereas the fifth assay improved from $96 \%$ to $98.5 \%$ accuracy and an $87 \%$ to $94 \%$ call rate. A second alternative is to redesign the assay. This is illustrated in Figure 3, which shows cluster plots for a TaqMan SNP assay before (Figure 3A) and after (Figure 3B) the addition of a single base to the $5^{\prime}$ probe end. This modification improves the FAM probe performance and leads to improved cluster separation.

DNA quality and amount per reaction is a third factor affecting SNP assay performance. Assay sensitivity to DNA amount in the nanotiter plate was evaluated by testing 32 pre-loaded TaqMan SNP assays against $48 \mathrm{CEPH}$ DNA templates at concentrations of $0.01-1 \mathrm{ng}$ per throughhole (Figure 4). The accuracy of each assay exhibits a different degree of sensitivity to the amount of DNA per reaction. Some assays maintain high accuracy essentially independent of the amount of DNA per reaction to as low as $0.1 \mathrm{ng}$ genomic human DNA (33 genomic equivalents), while others begin to lose accuracy rapidly at $<1 \mathrm{ng}$ genomic DNA ( $<330$ genomic equivalents). Commensurate with this loss of accuracy is an increase in heterozygote cluster variance that makes it more difficult to distinguish a heterozygote from a homozygote.

Samples with low DNA template per reaction can be genotyped in the nanotiter plates by first performing whole-genome amplification (WGA). Starting with a genomic DNA concentration of $3.2 \mathrm{ng} / \mu \mathrm{L}$ (0.1 ng per through-hole), amplification with the GenomiPhi V2 DNA Amplification Kit generated an average yield for 96 samples of $8.95 \mu \mathrm{g}$ per $20 \mu \mathrm{L}$ reaction, well above the stated typical yield $(4-7 \mu \mathrm{g})$. The WGA samples performed almost as well as the genomic DNA, exhibiting 99\% call rate and $100 \%$ concordance with the genotypes called with the genomic DNA samples (Figure 5).

\section{Discussion}

Single nucleotide polymorphism genotyping was demonstrated with the TaqMan biallelic PCR assay in a nanotiter plate fabricated as a high density array of fluidically isolated through-holes in a thin metal platen with surfaces differentially modified by hydrophilic and hydrophobic polymers. This nanofluidic device fluidically interfaces readily with microtiter plates to enable simple and efficient parallel transfer of liquid samples and PCR reagents into the nanoliter environment, resulting in a workflow for SNP genotyping large numbers (hundreds to thousands) of specimens with tens to hundreds of SNP TaqMan PCR assays per specimen. A key advantage of this system is the ability to simultaneously interrogate multiple samples per plate with multiple, independent genotype assays per sample. The independence of each assay in the panel means the assay can be changed for another 
without re-optimizing the entire assay panel, thus providing a high degree of flexibility in assay selection and future improvements to the assay panel.

The simple workflow, combined with batch thermal cycling of the nanotiter plates, can result in a high sustainable analytical throughput of up to 98,000 genotypes (64 SNPs per sample and 48 samples per nanotiter plate) per person per 8 -h shift with standard laboratory protocols and workflows. The nanotiter plate system fits on a standard laboratory bench, thereby eliminating the need for capital investment in robotic automation and the burden of its operating expense to achieve a high level of genotyping throughput.

Miniaturization of the TaqMan assays to a nanoliter format results in an assay performance similar to the same assay in a reaction volume $150 \times$ larger without requiring each assay to be individually optimized. Furthermore, for those assays whose performance does not translate directly into the nanoliter system, either duplicate genotype calling or a simple redesign of the TaqMan assay will recover the assay precision and accuracy. A clear benefit of assay miniaturization is the 64-fold decrease in TaqMan assay and PCR reagent consumption relative to standard $5-\mu \mathrm{L}$ TaqMan reactions in a microplate, resulting in an increase in the number of genotypes generated for the same amount of reagent consumed. Sample consumption can be decreased by over a factor of five based on the 1-20 ng per reaction recommended for each microplate TaqMan reaction (www3.appliedbiosystems. com/cms/groups/mcb_support/documents/ generaldocuments/cms_042998.pdf). Moreover, the nanotiter plate system can accommodate lower amounts of DNA per reaction without measurable loss of assay precision, by pre-amplification via wholegenome amplification prior to testing (29,30). For applications such as laser capture microdissection (31) that provide limited amounts of sample, this capability is likely to be particularly important. Together, these attributes make the nanotiter plate system well-suited for a number of SNP genotyping applications.

\section{Acknowledgments}

The authors wish to thank Kevin Papenfuss and Al Bodota for their technical assistance.

The authors are employed by BioTrove, Inc., the manufacturer of the OpenArray device and software described in this manuscript.

\section{References}

1. Matsuzaki, H., S. Dong, H. Loi, X. Di, G. Liu, E. Hubbell, J. Law, T. Berntsen, et al. 2004. Genotyping over 100,000 SNPs on a pair of oligonucleotide arrays. Nat. Methods 1:109-111.
2. Murray, S.S., A. Oliphant, R. Shen, C. McBride, R.J. Steeke, S.G. Shannon, T. Rubano, B.G. Kermani, et al. 2004. A highly informative SNP linkage panel for human genetic studies. Nat. Methods 1:113-117.

3. Shi, M.M. 2001. Enabling large-scale pharmacogenetic studies by high-throughput mutation detection and genotyping technologies. Clin. Chem. 47:164-172.

4. Kwok, P.-Y. and X. Chen. 1998. Detection of single nucleotide variations, p. 125-134. In J.K. Setlow (Ed.), Genetic Engineering, Vol. 20. Plenum Press, New York, NY, USA.

5. Saito, K., T. Nakayama, N. Sato, A. Morita, T. Takahashi, M. Soma, and R. Usami. 2006. Haplotypes of the plasminogen activator gene associated with ischemic stroke. Thromb. Haemost. 96:331-336.

6. Imayanitov, E.N., A.V. Togo, and K.P. Hanson. 2004. Searching for cancer-associated gene polymorphisms: promises and obstacles. Cancer Lett. 204:3-14.

7. Krejsa, C., M. Rogge, and W. Sadee. 2006. Protein therapeutics: new applications for pharmacogenetics. Nat. Rev. Drug Discov. 5:507-521

8. Efferth, T. and M. Volm. 2005. Pharmacogenetics for individualized cancer chemotherapy. Pharmacol. Ther. 107:155-176.

9. Denomme, G.A. and M. Van Oene. 2005. High-throughput multiplex single-nucleotide polymorphism analysis for red cell and platelet antigen genotypes. Transfusion 45:660-666.

10. Higgins, M., A. Hughes, N. Buzzacott, and J. Lown. 2004. High-throughput genotyping of human platelet antigens using the $5^{\prime}$-nuclease assay and minor groove binder probe technology. Vox Sang. 87:114-117.

11. Sobrino, B., M. Brion, and A. Carracedo. 2005. SNPs in forensic genetics: a review on SNP typing methodologies. Forensic Sci. Int. 154:181-194.

12. Smith, C.T., J. Baker, L. Park, L.W. Seeb, C. Elfstrom, S. Abe, and J.E. Seeb. 2005. Characterization of 13 single nucleotide polymorphism markers for chum salmon. Mol. Ecol. Notes 5:259-262.

13. Smith, C.T., W.D. Templin, J.E. Seeb, and L.W. Seeb. 2005. Single nucleotide polymorphisms provide rapid and accurate estimates of the proportions of U.S. and Canadian chinook salmon caught in Yukon river fisheries. N. Am. J. Fish. Manage. 25:944-953.

14. Giancola, S., H. McKhann, A. Berard, C. Camilleri, S. Durand, P. Libeau, F. Roux, X. Reboud, et al. 2006. Utilization of the three high-throughput SNP genotyping methods, the GOOD assay, Amplifluor and TaqMan, in diploid and polyploidy plants. Theor. Appl. Genet. 112:1115-1124.

15. Stephens, A.J., F. Huygens, J. Inman-Bamber, E.P. Price, G.R. Nimmo, J. Schooneveldt, W. Munckhof, and P.M. Giffard. 2006. Methicillin-resistant Staphylococcus aureus gentoytping using a small set of polymorphisms. J. Med. Microbiol. 55:43-51.

16. Koebner, R.M. and R.W. Summers. 2003. $21^{\mathrm{st}}$ century wheat breeding: plot selection or plate detection? Trends Biotechnol. 21:59-63.

17. Rafalski, A. 2002. Applications of single nucleotide polymorphisms in crop genetics. Curr. Opin. Plant Biol. 5:94-100.

18. Rohrer, G.A., B.A. Freking, and D. Nonneman. 2007. Single nucleotide polymor- phisms for pig identification and parentage exclusion. Anim. Genet. 38:253-258.

19. Tobler, A.R., S. Short, M.R. Andersen, T.M. Paner, J.C. Briggs, S.M. Lambert, P.P. Wu, Y. Wang, et al. 2005. The SNPlex Genotyping system: a flexible and scalable platform for SNP genotyping. J. Biomol. Tech. 16:398-406.

20. Jurinke, C., D. van den Boom, C. Cantor, and $\mathbf{H}$. Koster. 2001. Automated genotyping using the DNA MassArrayTM technology, p. 103-116. In J.B. Rampal,(Ed.), Methods in Molecular Biology, DNA Arrays: Methods and Protocols. Vol. 170. Humana Press Inc., Totowa, NJ, USA.

21. Livak, K.J. 1999. Allelic discrimination using fluorogenic probes and the $5^{\prime}$ nuclease assay. Genet. Anal. 14:143-149.

22. Zhang, C., X. Jinliang, W. Ma, and W.Zheng. 2006. PCR microfluidic devices for DNA amplification. Biotechnol. Adv. 24:243-284.

23. Ranade, K., M.-S. Chang, C.-T. Ting, D. Pei, C.-F. Hsiao, M. Olivier, R. Pesich, J. Hebert, et al. 2001. High-throughput genotyping with single nucleotide polymorphisms. Genome Res. 11:1262-1268

24. Morin, P.A., R. Saiz, and A. Monjazeb. 1999. High-throughput single nucleotide polymorphism genotyping by fluorescent $5^{\prime}$ exonuclease assay. BioTechniques 27:538-552.

25. Morrison, T., J. Hurley, J. Garcia, K. Yoder, A. Katz, D. Roberts, J. Cho, T. Kanigan, et al. 2006. Massively parallel, nanoliter, high throughput quantitative PCR. Nucleic Acids Res. 34:e123.

26. Brenan, C.J.H., K. Domansky, P. Kurzawski, and L.G. Griffith. 2000. BioMEMS applied to the development of cell-based bioassay systems. Proc. SPIE 3912:76-87.

27. Kanigan, T.S., C.J.H. Brenan, S. Lafontaine, L. Sosnowski, P.G. Madden, and I.W. Hunter. 2000. Living chips for drug discovery. Proc. SPIE 3926:172-180.

28. Brenan, C.J.H., T. Morrison, K. Stone, T. Heitner, A. Katz, T.S. Kanigan, R. Hess, S.-J. Kwon, et al. 2002. Massively parallel microfluidics platform for storage and ultrahigh-throughput screening. Proc. SPIE 4626:560-569.

29. Barker, D.L., M.S. Hansen, A.F. Faruqi, D. Giannola, O.R. Irsula, R.S. Lasken, M. Latterich, V. Makarov, et al. 2004. Two methods of whole-genome amplification enable accurate genotyping across a 2320-SNP linkage panel. Genome Res. 14:901-907.

30. Rook, M.S., S.M. Delach, G. Deyneko, A. Worlock, and J.L. Wolfe. 2004. Whole genome amplification of DNA from laser capture-microdissected tissue for high-throughput single nucleotide polymorphism and short tandem repeat genotyping. Am. J. Pathol. 164:23-33.

31. Emmert-Buck, M.R., R.F. Bonner, P.D. Smith, R.F. Chauaqui, Z. Zhuang, S.R. Goldstein, R.A. Weiss, and L.A. Liotta. 1996. Laser capture microdissection. Science 274:998-1001.

Received 26 September 2007; accepted 4 April 2008.

Address correspondence to Dr. Colin J.H. Brenan, BioTrove Inc., 12 Gill St., Ste. 4000 Woburn, MA 01810, USA. email: cbrenan@ biotrove.com 